protective factors of $\mathrm{CS}, \mathrm{BO}$ and compassion satisfaction (CS) amongst physicians in an Asian pediatric emergency department during a pandemic.

Methods A qualitative, individual interview methodology was employed. From March to April 2020, we enrolled 20 physicians involved in frontline care during the coronavirus disease 2019 (COVID-19) pandemic in the pediatric emergency department to participate. Semi-structured interviews were conducted, audiotaped and transcribed in verbatim, with identifiers removed. Themes were identified and data analysed using qualitative data analysis and iterative data analysis.

Results We recruited 20 physicians. Three themes emerged from data analysis. Firstly, we showed how workplace conditions are protective, including work morale, leadership and management and social support received. Secondly, workload affected participants emotionally and in terms of its heavier quantity and longer hours. Finally, intrinsic factors that were protective in developing $\mathrm{CF}$ or $\mathrm{BO}$ include having professional autonomy, experience, work-life balance and having emotional resilience to develop self-care. Cultural influences affect emotional regulation and can lead to negative coping with negative peer pressure. In general, participants quantified their levels of satisfaction at work as average to above-average. However, they highlighted experiencing greater stress during COVID-19 with the underlying fear of contagion and infection.

Conclusions Being a pediatric emergency physician puts one at greater risk of experiencing $\mathrm{CF}$ and $\mathrm{BO}$ because of work and non-work stressors especially during a global pandemic, influenced by sociocultural factors. A positive and supportive work environment should be created while providing culturallyadapted strategies to improve individual physician resilience to maintain their well-being

\section{CHALLENGES OF MONITORING AND TREATING RECURRENCE OF PRIMARY FOCAL SEGMENTAL GLOMERULOSCLEROSIS AFTER RENAL TRANSPLANTATION}

Christina Tran, Hilary Cramp. UK

\subsection{6/bmjpo-2021-RCPCH.11}

\section{Background}

Introduction In patients under 16 presenting with nephrotic syndrome, primary focal segmental glomerulosclerosis (FSGS) is an important differential. High recurrence rates following transplantation, and a considerable risk of progression to endstage renal disease, are well-recognised in cases with presumed circulating permeability factors rather than a genetic cause. To limit the risk of graft loss, it is therefore crucial to monitor for relapsing disease. The main marker of recurrence is proteinuria, however this can be challenging to interpret in a patient with high proteinuria pre-transplantation.

Objectives This is a case of a 14-year-old with FSGS, who developed early recurrence post-transplantation.

Methods

Case Presentation Pre-emptive renal transplantation was discussed with the family of a 14-year-old male with steroidresistant FSGS, who had also not responded to tacrolimus, mycophenolate and rituximab. Proteinuria would be difficult to interpret post-transplantation, as he continued to produce high levels of proteinuria with a significant urine output. A number of options were explored including; (1) bilateral nephrectomies or embolization pre-transplantation to shut down native function altogether; (2) a tube into the transplant renal pelvis passing through the bladder, serving as a catheter to monitor urine exclusively from the transplant kidney; (3) delay of transplantation until native kidney function completely ceased. Given the challenging practicality of (2), and uncertain timescale of (3), bilateral nephrectomies were arranged a few weeks prior to transplantation.

Results

Outcome After day five following a live donor kidney transplant, proteinuria developed progressively. Daily therapeutic plasma exchange was commenced for two weeks. Renal function continued to deteriorate, and lipoprotein apheresis was trialled. A transplant renal biopsy, nine days following lipoprotein apheresis, reported early recurrence of FSGS.

Conclusions

Discussion The leading cause of renal graft failure in primary FSGS, within the paediatric cohort, is disease recurrence. Removal of circulating permeability factors using extracorporeal systems, such as therapeutic plasma exchange and lipoprotein apheresis, could theoretically improve graft survival. Despite a number of possibilities considered, this case highlights the difficulties of monitoring and treating disease recurrence post-transplantation.

\section{DEVELOPMENT OF PERIOPERATIVE CHECKLIST FOR INFANTS REQUIRING SURGICAL INTERVENTION}

Vaibhav Katiyar, Theodore Dassios, Ravindra Bhat, Carolina Zorro, Ann Hickey. UK

\subsection{6/bmjpo-2021-RCPCH.12}

Background Infants requiring surgical intervention are at high risk of complications. Peri-operative checklists have demonstrated reduction in morbidity and mortality in many patient groups by maintaining high standards of care provided.

Objectives We audited compliance with current perioperative protocols on a surgical neonatal intensive care unit at King's College Hospital, London, UK and identified deficits in documentation and clinical practices. A perioperative checklist was then formulated as a quality improvement activity to standardize care which was re-audited to demonstrate significant improvisation of deficiencies.

Various new variables were introduced with the implementation of peri-operative check list to provide an extended and comprehensive health care package to infants undergoing surgical interventions. These included post-operative examination, IV antibiotics, replacement of ongoing fluid losses, identity bands and COVID swabs results prior to theatres.

Methods Review of case notes were undertaken pre and postoperatively. Documentation of clinical examination, laboratory investigations, imaging, ventilation plan, consent, communication, prescriptions of fluids, antibiotics and analgesia were assessed.

Results The case records of 48 infants (birth weight 460 4600 grams) with gestational ages ranging from 23-41 weeks were analysed (shown in table 1) both pre and post introduction of peri-operative checklist (shown in figure 1). The surgical interventions included laparotomy (32), neurosurgery (10), thoracotomy (4) and central venous line insertion (2). 